\title{
Independent imaging biomarkers do not exist
}

\author{
Harry B. Burke
}

Link to original article O'Connor, J. P. B. et al. Imaging biomarker roadmap for cancer studies. Nat. Rev. Clin. Oncol. 14, 169-186 (2017).

In their Consensus Statement published in the March 2017 issue of this journal (Imaging biomarker roadmap for cancer studies Nat. Rev. Clin. Oncol. 14, 169-186; 2017) $)^{1}$, James O'Connor and co-authors argue for the independent existence of 'imaging biomarkers'. They reject definitions of biomarkers that restrict "the scope of biomarkers to describing biological molecules" (REF. 1) and, instead, are guided by an FDA-NIH Biomarker Working Group consensus statement on biomarkers, which states that, "molecular, histologic, radiographic, or physiologic characteristics are examples of biomarkers" (REF. 2). Furthermore, O'Connor et al. suggest that the scope of biomarkers includes "both imaging biomarkers (IBs) and biospecimenderived biomarkers" (REF. 1). Finally, they argue that imaging biomarkers and biospecimen-derived biomarkers are clearly distinguishable: imaging biomarkers require imaging devices, they might require tracers or contrast agents, and "many IBs do not purport, even in principle, to measure any underlying analyte" (REF. 1).

The investigators begin their argument by setting up a 'straw-man' definition of biomarker - one that limits the term biomarker to biological molecules. Once the straw-man is knocked down as too restrictive, they expand the definition of biomarker to include molecular, histological, and physiological characteristics - all of which are biological descriptors. Accompanying their biological descriptors is the term 'radiographic', which, in this context, implies that radiographic images are of the same character as biological entities - which, of course, they are not.

A medical biomarker is defined by what it is and how it is used. A medical biomarker is any biological entity that can be used for medical prediction: for risk, diagnosis, or prognosis, including prevention and treatment. In other words, a medical biomarker is a subset of all biological entities - it is a biological entity that can be used for medical purposes, including prediction of risks, prevention, and treatment ${ }^{3}$.

What do we make of the argument that imaging biomarkers are distinct from biological biomarkers? I suggest that the authors have confused the detection method with what is being detected. Although the distinction between the imaging modality and what is being imaged has become blurred because of the inexact use of medical imaging terminology, these concepts are easily distinguishable. For example, if we image cancer tissue under an optical microscope, we would not say that we are observing an imaging biomarker. Rather, we would say that the tissue contains features that are biomarkers of cancer. Furthermore, this would be the case even if we stained the tissue. In other words, the role of imaging is to assist the observer in seeing biological features.

Assisted medical imaging, the presentation of visual information that cannot be acquired by the unaided eye, creates an image of a biological entity using the passive or active penetration or reflection of electromagnetic energy. The purpose of medical imaging is to improve our ability to visualize nonmalignant and malignant biological entities and processes and/or to visualize indices of these entities and processes. The confusion arises when a radiographic image is constructed (for example, by CT orMRI) and/or when a radiographic image is enhanced by an agent, such as contrast agents or radiolabelling. In these situations, one is tempted to think that the constructed and/or enhanced image is an independent reality when, in fact, it is simply a representation of the biomarker - one that improves our visualization of the biomarker.

In the future, this confusion will end because, in many situations, a viewable image will not exist. Biomarker data will be digitally acquired and stored, and computer-based expert systems will analyse the data and provide the relevant biomarker information. This nonvisual information will increase in the range and complexity of detected electromagnetic signals and it will substantially improve the clinical utility of biomarkers. Biomarkers will exist, but not as images.

In conclusion, although any biological entity can be an imaging target, medical biomarkers are imaged to assist in the care of patients. The imaging activity can include the construction of an image of the biomarker and/or the enhancement of an image of the biomarker, but the image is not the biomarker - the biological entity is the biomarker. The image is simply an improved visual representation of the biomarker. Independent imaging biomarkers do not exist.

Harry B. Burke is at the Section of Safety and Quality, Department of Medicine, F. Edward Hébert School of Medicine, Uniformed Services University of the Health Sciences, Building 53, Room 51,4301 Jones Bridge Road, Bethesda, Maryland 20814, USA.

harry.burke@usuhs.edu doi: $10.1038 /$ nrclinonc. 2017.83 Published online 13 Jun 2017

1. O'Connor, J. P. et al. Imaging biomarker roadmap for cancer studies. Nat. Rev. Clin. Oncol. 14, 169-186 (2017).

2. Food and Drug Administration $\&$ National Institutes of Health. BEST (Biomarkers, Endpoints, and other tools) resource. NCBI http://www.ncbi.nlm.nih.gov/books/ NBK326791 (2016).

3. Burke, H. B. Predicting clinical outcomes using molecular biomarkers. Biomark. Cancer 8, 89-99 (2016).

Competing interests statement

The author declares no competing interests. The views expressed by the author do not represent those of the US Government or of the Uniformed Services University of the Health Sciences. 